\title{
Michel Hastings, Loïc Nicolas, Cédric Passard (Dir.), Paradoxes de la transgression
}

Paris, CNRS Éditions, coll. « Philosophie et histoire des idées », 2012, $300 \mathrm{p}$.

\section{Daniel Vidal}

\section{(2) OpenEdition}

\section{Journals}

Édition électronique

URL : http://journals.openedition.org/assr/25508

DOI : $10.4000 /$ assr. 25508

ISSN : 1777-5825

Éditeur

Éditions de l'EHESS

Édition imprimée

Date de publication : 30 décembre 2013

Pagination : 215

ISSN : 0335-5985

\section{Référence électronique}

Daniel Vidal, « Michel Hastings, Loïc Nicolas, Cédric Passard (Dir.), Paradoxes de la transgression », Archives de sciences sociales des religions [En ligne], 164 | 2013, mis en ligne le 04 mars 2014, consulté le 21 septembre 2020. URL : http://journals.openedition.org/assr/25508 ; DOI : https://doi.org/ $10.4000 /$ assr. 25508

Ce document a été généré automatiquement le 21 septembre 2020.

(c) Archives de sciences sociales des religions 


\section{Michel Hastings, Loïc Nicolas, Cédric Passard (Dir.), Paradoxes de la transgression}

Paris, CNRS Éditions, coll. «Philosophie et histoire des idées », 2012, $300 \mathrm{p}$.

Daniel Vidal

\section{RÉFÉRENCE}

Michel Hastings, Loïc Nicolas, Cédric Passard (Dir.), Paradoxes de la transgression, Paris, CNRS Éditions, coll. « Philosophie et histoire des idées », 2012, 300 p. 
Comme toute notion utilisée et travaillée par les sciences sociales, la transgression ne définit pas une situation, ou une action, univoque. Disposée à juste titre en cet ouvrage au centre du jeu social, elle joue aux - et avec les - limites du tolérable, bouleverse frontières et normes, entretient un rapport ambivalent avec ce que toute société produit comme instance du sacré. Elle ne pose pas en permanence la question de la frontière entre ce qui est légitime et ce qui est interdit, sans aussitôt refonder limites et ordre social. Mais dès là apparaît une divergence dans la "fonction» de l'acte transgressif. Construction sociale de la limite sans cesse maintenue, parce que questionnée sans cesse par tel acte: la société en use comme sa plus belle chance de demeurer en passion d'ordre. Dans le

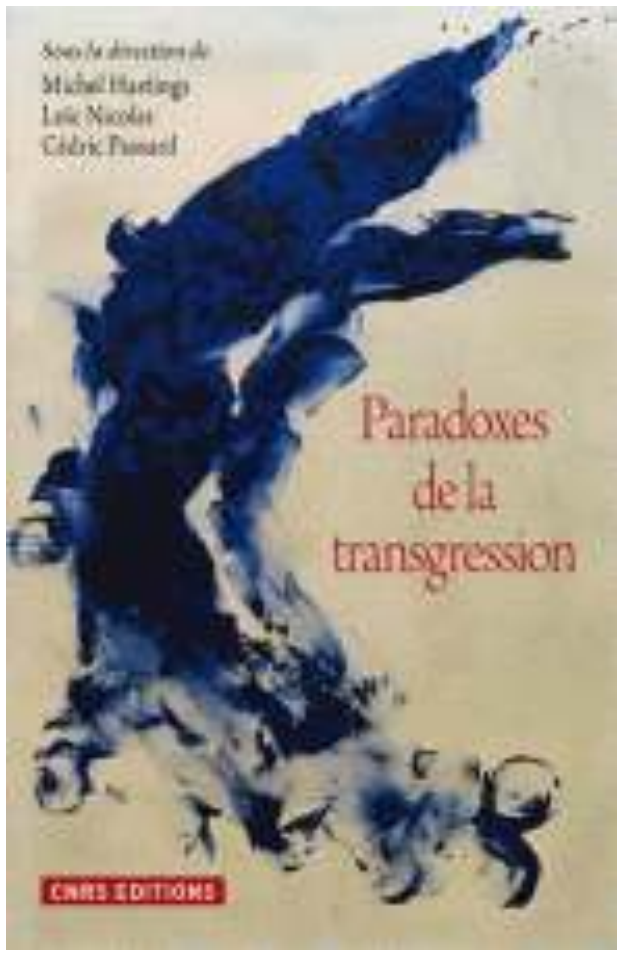
même mouvement, cependant, Georges

Bataille soulignait que s'il n'est pas, par la transgression, d'abolition de l'interdit, il n'est d'interdits que sous condition de son dépassement même. D'où cette tension irréductible de l'événement transgressif : réamorcer l'ordre au moment où il le brise ; demeurer scandale au moment où il refait société. Double profit, que les auteurs ne manquent pas de souligner: gagner un surcroît d'ordre par l'objection qui le transgresse - constituer l'interdit, et le sacré comme sa modalité la moins négociable, non plus comme cible, mais comme matrice même de toute transgression.

Dépassement de l'ordre social, la transgression est, selon l'expression de G. Balandier, « mise à mal radicale » de cet ordre. Plus grand est l'impératif d'ordre, plus nécessaire est le désordre somptuaire et maîtrisé. Les "maîtres du désordre » sont ceux-là trickster [tricheur], Legba le vodoun, clowm cérémoniel - qui poussent « la transgression au maximum de son extension » et, par sa ritualisation, recomposent un ordre. Toute transgression est chemin de liberté, avec le risque qui en est l'accompagnement obligé. On ne fréquente pas les zones sacrées - racines du pouvoir, raisons de créance - sans se mettre en jeu soi-même, en son intégrité corporelle ou mentale. Car il en va de la transgression comme de la réalisation d'un désir : «L'ordre est désiré, le désordre doit être fait pour qu'il y ait renforcement du désir d'ordre ». On ne peut dès lors ignorer de quel engagement personnel se paie la contribution transgressive à l'ordre social. Il y faut des individualités d'exception pour que soit rétabli le groupe en la maintenance banale de son existence collective. La désorceleuse du bocage, qu'a si magistralement étudiée Jeanne Favret-Saada, en peut être ici la figure capitale : elle fait récit du mal et bricole à son revers un récit improvisé/canonique de la guérison.

C'est à coup sûr l'inscription du sujet au principe de la transgression, qui me paraît donc constituer le fil rouge de cette interrogation plurielle. Quand déviance et délinquance sont qualifiées par Albert Ogien comme conduites non conformes, exigeant à toutes fins utiles que soient respectées les convenances d'un système social organisé, 
la transgression, inscrite également "dans le déroulement de l'activité pratique », se déploie en un au-delà d'une norme, faisant du même coup éclater la notion d'identité. "Simultanéité des identités », écrit A. Ogien. Mais surtout, et là me semble l'essentiel, acte transgressif assumé par le sujet comme délibéré, transparent à qui s'y livre, et, en quelque sorte, irrémissible. «Inexcusable », et acte singulier d'un « individu seul » venu à ce que l'on pourrait nommer sa souveraineté et la pleine conscience et responsabilité de son agir. Si la transgression est bien cet «acte qui vise ostensiblement et délibérément à choquer en brisant un tabou ", d'être une décision d'un seul, elle ne peut engendrer une sous-culture, sauf à se commettre comme geste à vocation politique explicite (déchaînement de barbarie, génocide, etc.). Mais on sort de la transgression pour entrer en infamie.

Pour bien marquer l'imputation personnelle à la racine de la transgression, cet « état émotionnel caractérisé d'abord par une ivresse de la surpuissance ", Philippe Braud propose qu'on la lise comme fondée en raison sur "la souveraineté d'un Moi", coupable dès lors, et assumé comme tel. "Forme audacieuse d'affirmation de soi ", elle est agence de liberté profanatrice et, par le délitement des frontières du sacré, fondatrice potentielle d'un ordre nouveau. Mais cela ne vaut que sous condition de définir l'Interdit comme "rigoureuse restriction de la liberté d'interprétation». Tout viol est alors immédiatement libérateur. Il est cependant, je l'ai évoqué, une autre lecture de la transgression, qui, loin de la penser comme ce qui porte atteinte à l'univers du sacré, fonde celui-ci en site propre du transgressif. Ce qui, avec Philippe Roussin, peut s'entendre, par exemple, de l'art en son versant tragique dionysiaque, dépense improductive parce que non référée à une économie marchande. Pure perte, selon R. Caillois et G. Bataille. Ainsi en va-t-il de la fête, de la guerre. De la poésie : s'il n'est plus de limite à la figuration ou à l'écriture (Nathalie Heinich qualifiera ainsi l'art contemporain, tout contenu et contenant dévergondé), c'est que le poète (ou l'artiste aujourd'hui) «dispose tout à coup seul de toutes les convulsions humaines qui sont possibles, et il ne peut pas se dérober devant cet héritage de la puissance divine - qui lui appartient ». C'est le sacré qui est instance de transgression. C'est donc aussitôt le profane qu'il convient de réenchanter. Telle est la condition de «l'individu moderne sans Dieu ».

Il était entendu, au XviI ${ }^{e}$ siècle, qu'il n'était de salut que dans l'excès. Affaire de mystique. S'il n'est pas aujourd'hui question de salut, est-il encore question d'excès ? La leçon emblématique de Bartleby peut valoir «figure limite de la privation radicale». " Je préfèrerais pas ", objecte-t-il à qui le somme d'agir. Faut-il encore qu'il soit présent au monde, quand il est au contraire en son avers, sinon son aversion. Son néant. Sa transgression. Homme "en train de s'inachever", selon la précieuse expression de Michel Hastings, qui s'appliquerait au grand œuvre de Pessoa. Sortir du monde, s'exiler du siècle : "trahir l'idéal de participation ", "être incrédule aux règles du jeu social ». Voici désenchantée la «figure du citoyen». Transgression capitale contre un monde qui tient le tout politique pour le principe même de l'ordre social. L'on peut alors, avec Loïc Nicolas, parler de "déclôture du sens", par la capacité à " composer, tailler et façonner des discours » à la façon des sophistes, dont l'art de la parole fait éclater les conventions langagières. Ainsi devient-il un instrument capable « d'arpenter le monde des possibles" par le travail même de l'argumentation. "Face à une parole sans souplesse et sans fragilité ", le sophiste, récusant toute inscription philosophique, « émancipe le sens » de ses contraintes normatives. Le monde proposé est alors tissu de précarités et d'incertitudes, et le rhétoricien, homme de nulle part. Où l'on retrouve 
l'économie de la déperdition des frontières et de la « déprime du sens » dont relèvent les événements transgressifs portés au plus haut degré de leur violence symbolique.

Il est des transgressions de pure stratégie "politique». Ainsi la trahison, ce viol de confiance et de loyauté qu'analyse Sébastien Schehr. Elle brise l'unité et l'homogénéité du groupe, en révélant ses failles intimes, proposant un sens immédiat à toute menace d'« inquiétante étrangeté ». Ainsi, selon Jean-Vincent Holeindre, la ruse, qui fait vaciller le rapport du vrai et $d u$ faux, perturbe les hiérarchies et les frontières du pensable. Quant au pamphlet, cette "escrime langagière" présentée par Cédric Passard, s'appropriant une parole d'exacte liberté, il use de la parole comme performance et mise en scène tragique, agissante par le seul fait d'être prononcée, et réceptacle d'émotions par la seule gravité de son verbe.

Questionner les limites du "tolérable» est, pour Christelle Reggiani, s'interroger sur les ruptures linguistiques et esthétiques expérimentées par des acteurs majeurs de notre "modernité » littéraire. Hugo, bien sûr, mettant "un bonnet rouge au vieux dictionnaire ", et Céline, cela va de soi, ou Guyotat. Et l'OuLiPo (Queneau, Pérec, Roubaud), ce laboratoire qui voulut mettre l'écriture au défi de la mathématique. Soit trouver l'équivalent littéraire « d'un texte mathématique formalisable selon la méthode axiomatique ». Transgresser l'usage normé des mots en les soumettant à des contraintes autrement plus exigeantes que simplement syntaxiques ou grammaticales. Renoncer « à l'héroïsme individuel ou collectif de la narration moderne », pour accéder à une écriture qui soit une "harmonie» contre «la contingence calamiteuse de l'histoire ». L'écriture contre le désastre : une écriture enfin venue à sa raison, qui est son nombre. À l'autre extrémité du spectre transgressif, la pornographie. L'acte, sans doute, mais, plus encore, sa représentation. Il convient, écrit Ruwen Ogien, de distinguer très rigoureusement ce qui relève de l'offense (faite aux « bonnes mœurs ») et le préjudice (dommage grave porté à quiconque). De l'offense, on ne peut, on ne doit, rien en conclure sur le plan moral : ceci est affaire de jugement personnel. Le préjudice seul peut être soumis au jugement « civil » et faire l'objet de sanction. Les frontières du tolérable sont ainsi qualifiées à nouveau frais : «ce que nous faisons de nos propres vies, tant que nous ne causons pas de tort à autrui, n'a pas d'importance morale ». Ainsi R. Ogien peut-il proposer une "éthique minimale ", qui excepte de tout interdit ce qui relève de la seule morale personnelle, sous réserve que nul préjudice public n'en vienne dévoyer l'accomplissement. Sans la liberté d'offenser, il n'est pas de transgression possible. L'ordre social dépend très précisément de cette capacité à admettre le déploiement d'un désordre et dérèglement généralisé de toutes les instances et normes, afin que soit éprouvée en permanence sa capacité à réengendrer sa formule et sa loi. Ultime paradoxe, en effet, de la transgression. 